\title{
Phase diagrams of spin-3/2 Blume-Capel model on rectangular lattice under longitudinal magnetic field
}

\author{
O. Baran*, R. Levitskii \\ Institute for Condensed Matter Physics of the National Academy of Sciences of Ukraine, \\ 1 Svientsitskii Str., 79011 L'viv, Ukraine
}

\begin{abstract}
The spin-3/2 Blume-Capel model on a rectangular lattice with the ferromagnetic bilinear short-range interaction $K^{\mathrm{F}}=K(1+x)$ in one direction and the anti-ferromagnetic one $K^{\mathrm{AF}}=K(-1+x)$ in the perpendicular direction under a longitudinal magnetic field and in the presence of a single-ion anisotropy is investigated within the mean field approximation. The quantity $x$ characterizes coupling between the interactions in the perpendicular directions and its absolute value does not exceed unity. Basing on the study of temperature dependencies of sublattice magnetizations the phase diagrams in the $(x$, temperature) plane are constructed for different values of the longitudinal magnetic field and the single-ion anisotropy. The global phase diagram of the model demonstrates an unusual abundance.
\end{abstract}

Keywords: spin-3/2, Blume-Capel model, longitudinal field, mean field approximation

\footnotetext{
${ }^{*}$ Corresponding author

Email address: ost@icmp.lviv.ua (O. Baran)
} 


\section{Introduction}

The Ising models with spin $S>1 / 2$ and their numerous variations have been extensively studied during recent years due to a relative simplicity with which calculations for these models within different techniques can be carried out for testing of these methods as well as the theoretical interest arising from the richness of the phase diagrams due to competition of models parameters [1-12]. On the other hand, most modifications of the Ising model are applied for description of real objects. Namely, the spin-3/2 BlumeEmery-Griffiths model with nearest-neighbor interactions, both bilinear and biquadratic, and a single-ion anisotropy (or a crystal-field interaction) was introduced to explain the phase transition in $\mathrm{DyVO}_{4}$ qualitatively [13] and proved to be useful for description tricritical properties of ternary fluid mix-

tures [14]. The spin-3/2 Blume-Capel model, which is the partial case of the spin-3/2 Blume-Emery-Griffiths model without biquadratic interactions, can be applied to study $\mathrm{KEr}\left(\mathrm{MoO}_{4}\right)_{2}[15,16]$.

Theoretical techniques used to investigate the spin-3/2 Ising models include the mean field approximation (MFA) [4, 11 14, 17, 18], the effective field theory [19, 20], the cluster approximation as well as the Bethe approximation (the exact results for Bethe lattices) [9, 10, 21], the renormalizationgroup method [22], the transfer-matrix finite-size-scaling calculations [5]. These models have also been studied by means of the Monte-Carlo simulations [5, 18, 23].

Most of the papers, where the spin-3/2 Ising models are investigated, consider the lattices with either ferromagnetic bilinear interactions or anti- 
ferromagnetic ones. In this work we study the spin-3/2 Blume-Capel model

$$
H=-\sum_{i=1}^{L} \sum_{j=1}^{L}\left[\Gamma S_{i, j}+D S_{i, j}^{2}+K^{\mathrm{F}} S_{i, j} S_{i+1, j}+K^{\mathrm{AF}} S_{i, j} S_{i, j+1}\right]
$$

on the rectangular lattice with the ferromagnetic bilinear short-range interaction $K^{\mathrm{F}}=K(1+x)$ in one direction and the antiferromagnetic one $K^{\mathrm{AF}}=K(-1+x)$ in the perpendicular direction, where $\Gamma$ is a longitudinal magnetic field, $D$ is a single-ion anisotropy, $K>0, x \in]-1,1[$. Each $S_{i, j}=S_{i, j}^{z}$ takes the value $\pm 1 / 2$ or $\pm 3 / 2$. We will especially investigate the effect of swapping of interactions strength $x$ within the MFA.

\section{Mean field approximation}

The expression for a free energy of the Blume-Capel model (11) within the MFA is constructed on the basis of the single-site Hamiltonians

$$
\begin{aligned}
& H_{i_{a}}=-\varkappa_{a} S_{i_{a}}-D S_{i_{a}}^{2}, \\
& \varkappa_{a}=\Gamma+2 K^{\mathrm{F}} m_{a}+2 K^{\mathrm{AF}} m_{b}, \quad m_{a}=\left\langle S_{i_{a}}\right\rangle, \quad(a, b=A, B)
\end{aligned}
$$

(where $A$ and $B$ refer to two different sublattices) in a usual way [17, 24 26] as follows:

$$
\begin{aligned}
& F=-\frac{N}{2} k_{\mathrm{B}} T\left[\ln Z_{1_{A}}+\ln Z_{1_{B}}\right]+\frac{N}{2} K^{\mathrm{F}}\left(m_{A}^{2}+m_{B}^{2}\right)+N K^{\mathrm{AF}} m_{A} m_{B} \\
& Z_{1_{a}}=2 \mathrm{e}^{\beta D / 4}\left[\operatorname{ch}\left(\beta \varkappa_{a} / 2\right)+\mathrm{e}^{2 \beta D} \operatorname{ch}\left(3 \beta \varkappa_{a} / 2\right)\right] .
\end{aligned}
$$

Here $\beta=\left(k_{\mathrm{B}} T\right)^{-1}$, and $N=L^{2}$ is the total number of spins.

Within the MFA the magnetizations of sublattices $m_{A}$ and $m_{B}$ can be determined [16, 17, 24 27] from the conditions of extremum of the free energy 
with respect to them $\left(\partial F / \partial m_{A}=\partial F / \partial m_{B}=0\right)$. These conditions yield the following system of equations for $m_{a}$ :

$$
\begin{aligned}
& \mathrm{e}^{\beta D / 4}\left[\operatorname{sh}\left(\beta \varkappa_{A} / 2\right)+3 \mathrm{e}^{2 \beta D} \operatorname{sh}\left(3 \beta \varkappa_{A} / 2\right)\right] / Z_{1_{A}}-m_{A}=0, \\
& \mathrm{e}^{\beta D / 4}\left[\operatorname{sh}\left(\beta \varkappa_{B} / 2\right)+3 \mathrm{e}^{2 \beta D} \operatorname{sh}\left(3 \beta \varkappa_{B} / 2\right)\right] / Z_{1_{B}}-m_{B}=0 .
\end{aligned}
$$

\section{Numerical analysis results}

Let us consider results of our numerical investigation of model (11) within the MFA.

To gain a better insight into the following results, let us explain principles of construction of phase diagrams and classification of phases. At given values of model parameters $K, x$ (i.e. fixed values of $K^{\mathrm{F}}=K(1+x)$ and $\left.K^{\mathrm{AF}}=K(-1+x)\right), \Gamma$, and $D$ we study temperature dependencies of solutions of the equation set ([6). The number of these solutions (i.e. pairs of temperature dependent branches of solutions) varies at different values of model parameters. Some pairs of branches-solutions exist on the whole temperature interval $T \in[0, \infty[$, others are defined on the limited intervals starting from the zero temperature $T \in\left[0, T_{1}\right]$, or the non-zero one $T \in\left[T_{2}, T_{3}\right]$. Choosing pairs of solutions which correspond to the absolute minimum of the free energy, we obtain temperature dependencies for magnetizations of sublattices. Calculating in this manner sublattice magnetizations for ranges of values of model parameters we construct phase diagrams for the considered model.

At temperature of the first order phase transition magnetizations of sublattices "jump" from one pair of branches-solutions of the equation set (6) 
to other one. Thus, different thermodynamically stable pairs of branchessolutions correspond to different phases (on the respective temperature intervals). Names of the ordered phases include values of solutions at the zero temperature which corresponds to the branches-solutions describing sublattice magnetizations in the chosen phase.

Thus we shall distinguish the following phases (see [4]):

- disordered phase $(\mathbf{d})$;

- antiferrimagnetic phase with $m_{A}=-3 / 2, m_{B}=3 / 2$ at $T=0\left(\mathbf{a i}_{-3 / 2,+3 / 2}\right)$;

- antiferrimagnetic phase with $m_{A}=-1 / 2, m_{B}=3 / 2$ at $T=0\left(\mathbf{a i}_{-1 / 2,+3 / 2}\right)$;

- antiferrimagnetic phase with $m_{A}=-1 / 2, m_{B}=1 / 2$ at $T=0\left(\mathbf{a i}_{-1 / 2,+1 / 2}\right)$;

- ferrimagnetic phase with $m_{A}=1 / 2, m_{B}=3 / 2$ at $T=0\left(\mathbf{i}_{+1 / 2,+3 / 2}\right)$.

However, in the considered system there are also ordered phases where sublattice magnetizations are defined by the branches-solutions starting from non-zero temperatures. Such phases are simply designed as ferrimagnetic (i) or antiferromagnetic (ai) without indices describing ground state magnetizations.

This simple and clear classification of phases by indices, corresponding to the magnetizations in the ground state, is convenient for investigation of temperature dependencies of sublattice magnetizations because at $T=0$ magnetizations reach their asymptotical values (i.e. $m_{A}=-3 / 2, m_{B}=$ $3 / 2$, or $m_{A}=-1 / 2, m_{B}=3 / 2$, or $m_{A}=-1 / 2, m_{B}=1 / 2$, or $m_{A}=$ $\left.1 / 2, m_{B}=3 / 2\right)$. However, there are also phase transitions between ordered phases at variation of the $x$ parameter. While studying $x$-dependencies of magnetizations at $T>0$, such ordered phases can not be designed by the same indices as we do it considering temperature dependencies. 
Let us also note that within MFA the phase transitions between different ordered phases can be only of the first order and the transitions between ordered and disordered phases can be both of the second and of the first order.

At first, changes in topologies of $T$ vs $x$ phase diagrams at variation of the single-ion anisotropy are studied for the case $\Gamma / K=1$. Representative $T$ versus $x$ phase diagram cross-sections for different values of the single-ion anisotropy $D$ of model (1) at $\Gamma / K=1$ are presented in Figs. 115.

If the value of the single-ion anisotropy is large, the topology of the $(x, T)$ phase diagrams within MFA is the same as of the diagram given in Fig. 1 (where $D / K=20$ ). There are the second order and the first order transition lines separating the disordered phase from the antiferrimagnetic phase $\mathbf{a i}_{-3 / 2,+3 / 2}$. These lines join together at the tricritical point (TCP).

In the case $D / K=-1.65$ (see Fig. 21) the phase diagram has topology with a tricritical point, a triple point (TP) and a critical point (CP) inside the ordered phase. In this case the MFA predicts two antiferrimagnetic phases $\mathbf{a} \mathbf{i}_{-3 / 2,+3 / 2}$, and $\mathbf{a} \mathbf{i}_{-1 / 2,+3 / 2}$. The phase transition $\mathbf{a i}_{-3 / 2,+3 / 2} \rightarrow \mathbf{d}$ can be of the first or the second order, but the phase transitions $\mathbf{a} \mathbf{i}_{-3 / 2,+3 / 2} \rightarrow \mathbf{a i}_{-1 / 2,+3 / 2}$ and $\mathbf{a} \mathbf{i}_{-1 / 2,+3 / 2} \rightarrow \mathbf{d}$ are of the first order only.

When $D / K=-1.665$ there are three different ordered phases in the system: two antiferrimagnetic phases $\mathbf{a} \mathbf{i}_{-3 / 2,+3 / 2}, \mathbf{a i}_{-1 / 2,+3 / 2}$ and the ferrimagnetic one $\mathbf{i}_{1 / 2,+3 / 2}$. The phase diagram (see Fig. 3) has the topology with two tricritical points, two triple points, a critical end point (CEP), a critical point inside the ordered phase, and a re-entrant region. The sequence of temperature phase transitions $\mathbf{d} \rightarrow \mathbf{i}_{+1 / 2,+3 / 2} \rightarrow \mathbf{d}$ is possible (at 
$x \in[0.666112,0.6661157])$.

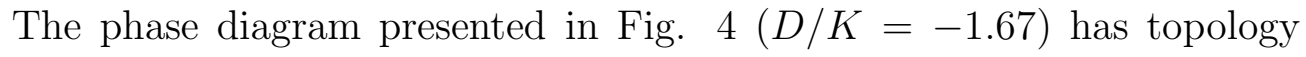
with two tricritical points, a critical end point, a critical point inside the ordered phase, and a double re-entrant region. At $x \in[0.66799999,0.668]$ the cascade of phase transitions $\mathbf{i}_{+1 / 2,+3 / 2} \rightarrow \mathbf{d} \rightarrow \mathbf{i}_{+1 / 2,+3 / 2} \rightarrow \mathbf{d}$ is possible. At $x \in[0.668,0.66809]$ the system undergoes the sequence of temperature phase transitions $\mathbf{d} \rightarrow \mathbf{i}_{+1 / 2,+3 / 2} \rightarrow \mathbf{d}$.

In the case $D / K=-1.8$ the $x$ vs temperature phase diagram contains a tricritical point and two critical points inside the ordered phase (see Fig. 55).

Topologies of $(x, T)$ phase diagrams at $D / K=-1.879$ and $D / K=-1.89$ shown in Figs. 6] and 7 differ from the described above ones by the presence of two additional tricritical points. Moreover at $D / K=-1.89$ the first order phase transition line has a minimum at $x \approx 0.59$ and the line of critical points (the second order phase transition line) has a maximum at $x \approx 0.64$.

At $D / K=-1.95$ the phase diagram contains three tricritical points (as well as in the case $D / K=-1.89)$, only one critical point inside the ordered phase and a triple point (instead of the second critical point; see Figs. 7 and 8).

In the case $D / K=-1.97$ (see Fig. 9) comparing to the described above cases there is an additional ordered phase: antiferrimagnetic $\mathbf{a i}_{-1 / 2,+1 / 2}$ phase. The $(x, T)$ phase diagram at $D / K=-1.97$ has topology with three tricritical points, a triple point and two critical point inside the ordered phase.

The phase diagram at $D / K=-1.99$ shown in Fig. 10 has topology with three tricritical points, two triple points, a critical point inside the ordered 
phase and a re-entrant region. The cascade of temperature phase transitions $\mathbf{a i}_{-1 / 2,+1 / 2} \rightarrow \mathbf{a i}_{-1 / 2,+3 / 2} \rightarrow \mathbf{a i}_{-1 / 2,+1 / 2}$ is possible at $x \in[-0.03651,0.0336]$.

In the case $D / K=-1.9999$ (see Fig. 11) the phase diagram has topology with three tricritical points, three triple points and two re-entrant regions. At $x \in[-0.0002115,0.0002015]$ and at $x \in[0.0000213,0.0000415]$ sequences of temperature phase transitions $\mathbf{a} \mathbf{i}_{-1 / 2,+1 / 2} \rightarrow \mathbf{a i}_{-1 / 2,+3 / 2} \rightarrow \mathbf{a i}_{-1 / 2,+1 / 2}$ and $\mathbf{d} \rightarrow \mathbf{a i}_{-1 / 2,+1 / 2} \rightarrow \mathbf{d}$ are possible, respectively.

At $D / K=-2.1,-2.4$ and -2.49 there are two different ordered phases in the system: antiferrimagnetic $\mathbf{a i}_{-1 / 2,+1 / 2}$ and ferrimagnetic $\mathbf{i}_{+1 / 2,+3 / 2}$. The $(x, T)$ phase diagrams presented in Figs. 12 and 13 contain three tricritical points while the diagram presented in Fig. 14 contains only one tricritical point. When $D / K=-2.4$ and -2.49 the MFA predicts also the double re-entrant temperature phase transitions $\mathbf{i}_{+1 / 2,+3 / 2} \rightarrow \mathbf{d} \rightarrow \mathbf{i}_{+1 / 2,+3 / 2} \rightarrow \mathbf{d}$ at $x \in[0.95994,0.96]$ and $x \in[0.995999,0.996]$, respectively. The re-entrant transitions $\mathbf{d} \rightarrow \mathbf{i}_{+1 / 2,+3 / 2} \rightarrow \mathbf{d}$ occur for $D / K=-2.4$ at $\left.\left.x \in\right] 0.96,0.96055\right]$ and for $D / K=-2.49$ at $x \in] 0.996,1[$.

At a small value of the single-ion anisotropy, the topology of the $(x, T)$ phase diagrams within MFA is the same as of the diagram given in Fig. 15 (when $D / K=-3$ ). Disordered and antiferrimagnetic $\mathbf{a i}_{-1 / 2,+1 / 2}$ phases are separated by the lines of phase transition both of the first and the second orders.

It is obvious, that the described above sequence of different topologies of $(x, T)$ phase diagrams under change of the single-ion anisotropy takes place only at $\Gamma \approx 1$. Other values of the longitudinal field give essentially different pictures as follows from our investigation of topologies of $(x, T)$ phase dia- 
grams with variation of the longitudinal field at a fixed value of the single-ion anisotropy. The most interesting phase diagrams at $D / K=-1.5$ and different values of $\Gamma$ are presented below. They demonstrate principal changes of topologies of phase diagrams caused by the field variation at $D / K \approx-1.5$.

In the case of the zero longitudinal field, the MFA results for thermodynamical characteristics of model (11) do not depend on $x$ [17]. At $k_{\mathrm{B}} T / K=3.296$ and any $\left.x \in\right]-1,1[$ (see Fig. [16) the system undergoes the second order temperature phase transition from the antiferromagnetic af $_{-3 / 2,+3 / 2}$ phase [4, 17] to the paramagnetic phase.

If the value of the longitudinal field is small, the topology of the $(x, T)$ phase diagram is the same as of the diagrams given in Fig. 16 for $\Gamma / K=0.1$ and $\Gamma / K=1$. Thick and thin solid lines in these phase diagrams represent, respectively, the second order and the first order transition lines from the antiferrimagnetic $\mathbf{a i}_{-3 / 2,+3 / 2}$ phase to the disordered phase. It should be noted that the topology of two phase diagrams described above is the same as the topology of the diagram presented in Fig. 1 in the first part of our study (where the influence of single-ion anisotropy on $(x, T)$ diagrams is investigated).

In the case $\Gamma / K=1.45$ (see Fig. 17) the phase diagram contains tricritical, triple and critical points, similarly to the case presented in Fig. 2.

The $(x, T)$ phase diagram at $\Gamma / K=1.5$ (Fig. 18) has topology with a tricritical point, a critical point inside the ordered phase, three triple points and a re-entrant region. The cascade of temperature phase transitions $\mathbf{d} \rightarrow$ $\mathbf{i}_{+1 / 2,+3 / 2} \rightarrow \mathbf{d}$ is possible (at $x \in[0.5,0.500000014]$ ).

At $\Gamma / K=1.6$ (Fig. 19) the phase diagram contains also a tricritical 
point, a critical point inside the ordered phase, but only one triple point in comparison with three TPs in the described above case.

It should be noted that there is a certain similarity between diagrams presented in Figs. 3, 4 and in Figs. 18, 19, respectively. The diagrams shown in Figs. 18 and 19 contain only single tricritical points. Moreover, they contain triple points instead of critical end points present in Figs. 3 and 4.

In the case $\Gamma / K=2.5$ (see Fig. 201), similarly to the case presented in Fig. 5, the phase diagram contains two critical points inside the ordered phase and a single tricritical point.

The $T$ vs $x$ phase diagram at $\Gamma / K=5$ (Fig. 21) has topology with a tricritical point, only one critical point, re-entrant and double re-entrant regions. At $x \in[-0.66666675,-0.66666669]$ and $x \in[-0.20662,-0.20623]$ the cascades of temperature phase transitions $\mathbf{a i}_{-1 / 2,+3 / 2} \rightarrow \mathbf{i}_{+1 / 2,+3 / 2} \rightarrow$ $\mathbf{a i}_{-1 / 2,+3 / 2}$ and $\mathbf{i}_{+1 / 2,+3 / 2} \rightarrow \mathbf{d} \rightarrow \mathbf{i}_{+1 / 2,+3 / 2} \rightarrow \mathbf{d}$ are possible, respectively. The middle phase transition in the sequence $\mathbf{i}_{+1 / 2,+3 / 2} \rightarrow \mathbf{d} \rightarrow \mathbf{i}_{+1 / 2,+3 / 2} \rightarrow \mathbf{d}$ can be of the first or the second order.

The topology of the diagram at $\Gamma / K=5.5$ shown in Fig. 22 differs from the described above one by the fact that the tricritical point (see Fig. 21) splits into a critical end point and a critical point inside the ordered phase. Moreover, there are two additional ferrimagnetic phases with sublattice magnetizations which correspond to branches (solutions of the equation set (6) ) starting from the non-zero temperature. As mentioned above, such phases are simply designated as ferrimagnetic $\left(\mathbf{i}^{(1)}\right.$ and $\left.\mathbf{i}^{(2)}\right)$ without indices describing ground state magnetizations. 
Besides the above mentioned special points (two CP and one CEP) on the phase diagram there is another "special" point (SP) at $x_{\mathrm{SP}}=-0.302678$, located between CEP (at $\left.x_{\mathrm{CEP}}=-0.302735\right)$ and CP (at $\left.x_{\mathrm{CP}}=-0.302594\right)$ in the curve of the first order phase transition. The type of the temperature phase transition changes from $\mathbf{i}^{(1)} \rightarrow \mathbf{i}_{+1 / 2,+3 / 2}$ to $\mathbf{i}^{(1)} \rightarrow \mathbf{i}^{(2)}$ at this SP. For example, at $x=-0.3027$ the system undergoes the phase transitions $\mathbf{i}_{+1 / 2,+3 / 2} \rightarrow \mathbf{d} \rightarrow \mathbf{i}^{(1)} \rightarrow \mathbf{i}_{+1 / 2,+3 / 2} \rightarrow \mathbf{d}$ at increase of temperature; but at $x=-0.3026$ the system undergoes the transitions $\mathbf{i}_{+1 / 2,+3 / 2} \rightarrow \mathbf{d} \rightarrow \mathbf{i}^{(1)} \rightarrow$ $\mathbf{i}^{(2)} \rightarrow \mathbf{d}$.

It should be pointed out that the phase diagram in Fig. 22 is complete. The above mentioned SP does not originate any additional curve of phase transition, what can be proved supplementing the study of temperature dependencies at fixed $x$ by the investigation of $x$-dependencies of $m_{A}$ and $m_{B}$ at fixed temperatures. Variation of $x$ in the vicinity of $x_{\mathrm{SP}}$ does not indicate any phase transition at $T=T_{\mathrm{SP}}+\varepsilon(\varepsilon \rightarrow 0)$.

Such a definition of SP as in Fig. 22 is useful for our study of temperature dependencies of magnetizations and the adopted above classification of phases.

At $x$ larger then $x_{\mathrm{CP}}=-0.302594$ the difference between phases $\mathbf{i}^{(1)}$ and $\mathbf{i}^{(2)}$ disappear as it usually happens above the critical point. This combined phase is designated as $\mathbf{i}^{(1 \mid 2)}$. Thus, for example, at $x=-0.301$ the system undergoes the phase transitions $\mathbf{i}_{+1 / 2,+3 / 2} \rightarrow \mathbf{d} \rightarrow \mathbf{i}^{(1 \mid 2)} \rightarrow \mathbf{d}$ at increase of temperature.

Increase of the longitudinal field shifts curves of phase transition in $(x, T)$ phase diagrams to the left (see Figs. 22 - 24). For example, the phase 
$\mathbf{i}_{-1 / 2,+3 / 2}$ is already absent in the phase diagram at $\Gamma / K=6.7$ (Fig. 23) as compared with the phase diagram at $\Gamma / K=5.5$ (Fig. 22) and there are only two phases (disordered and $\mathbf{i}^{(1 \mid 2)}$ ) in the phase diagram at $\Gamma / K=9.1$ (Fig. 24).

It should be mentioned, that $\mathrm{SP}$ at $x_{\mathrm{SP}}=-0.5401103$ almost coincides with the critical point at $x_{\mathrm{CP}}=-0.5401101$ in the $(x, T)$ phase diagram at $\Gamma / K=6.7$. At $x<-0.540725$ (see Fig. 23) only one temperature phase transition $\mathbf{i}_{+1 / 2,+3 / 2} \rightarrow \mathbf{d}$ is possible. At $\left.x \in\right]-0.540725, x_{\mathrm{CEP}}[$ (where $\left.x_{\mathrm{CEP}}=-0.54055\right)$ the MFA yields the sequence of transitions $\mathbf{i}_{+1 / 2,+3 / 2} \rightarrow$ $\mathbf{d} \rightarrow \mathbf{i}_{+1 / 2,+3 / 2} \rightarrow \mathbf{d}$. At $\left.x \in\right] x_{\mathrm{CEP}}, x_{\mathrm{SP}}[$ the system undergoes the phase transitions $\mathbf{i}_{+1 / 2,+3 / 2} \rightarrow \mathbf{d} \rightarrow \mathbf{i}^{(1)} \rightarrow \mathbf{i}_{+1 / 2,+3 / 2} \rightarrow \mathbf{d}$ at increase of temperature. At $x \in] x_{\mathrm{SP}}, x_{\mathrm{CP}}\left[\right.$ the system undergoes the phase transitions $\mathbf{i}_{+1 / 2,+3 / 2} \rightarrow \mathbf{d} \rightarrow$ $\mathbf{i}^{(1)} \rightarrow \mathbf{i}^{(2)} \rightarrow \mathbf{d}$. At $\left.x \in\right] x_{\mathrm{CP}},-0.54[$ and at $x \in]-0.54,-0.51777[$ there are the cascades of temperature phase transitions $\mathbf{i}_{+1 / 2,+3 / 2} \rightarrow \mathbf{d} \rightarrow \mathbf{i}^{(1 \mid 2)} \rightarrow \mathbf{d}$ and $\mathbf{d} \rightarrow \mathbf{i}^{(1 \mid 2)} \rightarrow \mathbf{d}$, respectively.

\section{Conclusions}

The spin-3/2 Blume-Capel model on the rectangular lattice, in which the short-range bilinear interactions in perpendicular directions are of opposite signs, is studied within the mean field approximation. The temperature vs $x$ phase diagrams ( $x$ characterizes coupling between the bilinear shortrange interactions in the perpendicular directions) at $\Gamma / K=1$ and different values of the single-ion anisotropy as well as at $D / K=-1.5$ and different values of the longitudinal magnetic field are obtained. The phase diagrams

presented in this paper illustrate abundance of the global phase diagram of 
the considered model.

It has been shown that the usual classification of phases (like used in works $[4$, 5, $10-12,18,19,22,23])$ which distinguishes phases $\mathbf{a i}_{-3 / 2,+3 / 2}, \mathbf{a i}_{-1 / 2,+3 / 2}$, $\mathbf{a i}_{-1 / 2,+1 / 2}, \mathbf{i}_{+1 / 2,+3 / 2}$ (where indices correspond to sublattice magnetizations in the ground state) is mainly suitable for investigation of temperature dependencies of sublattice magnetizations. However, a different class of ordered phases is found at the certain values of model parameters which does not easily fit the mentioned classification. These phases exist in the temperature intervals starting from the non-zero temperatures. 


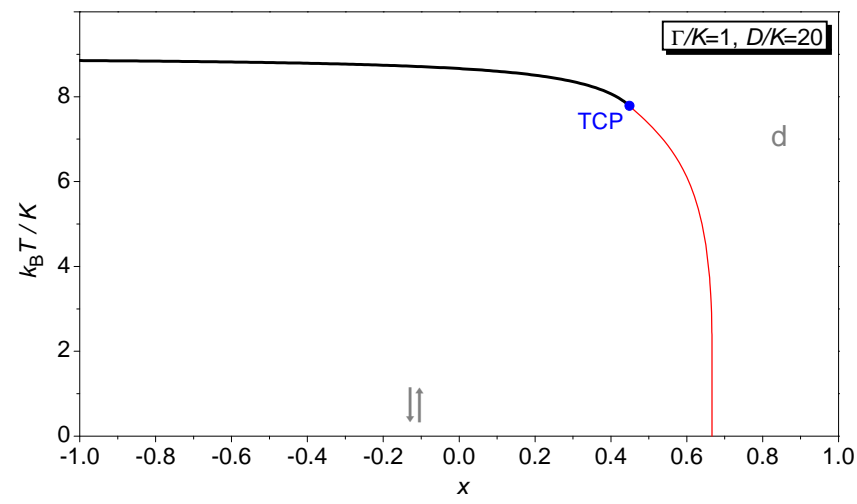

Figure 1: The $T$ vs $x$ phase diagram at $D / K=20$ and $\Gamma / K=1$. Thick and thin lines correspond to the phase transition between ordered and disordered phases of the second and the first order, respectively. The special point is a tricritical point.

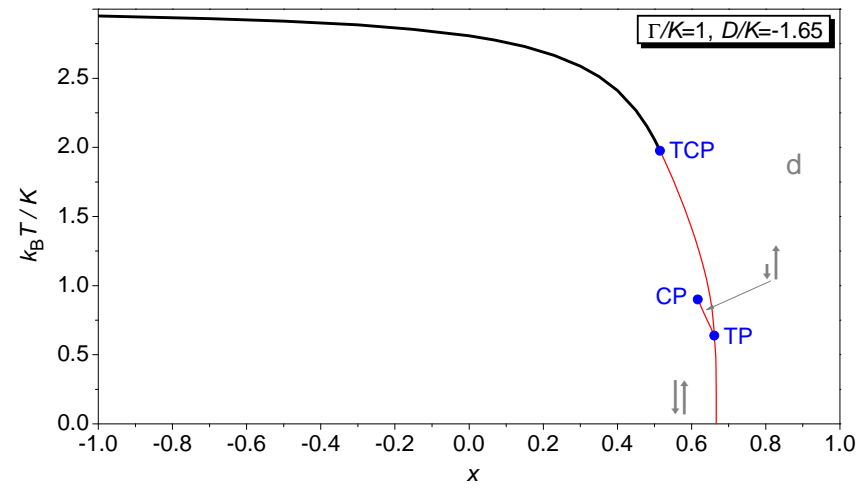

Figure 2: The $T$ vs $x$ phase diagram at $D / K=-1.65$ and $\Gamma / K=1$. A thick line indicates the phase transition between ordered and disordered phases of the second order. Thin lines indicate the first order phase transitions between ordered and disordered phases as well as between different ordered phases. The special points are a tricritical point, a critical point and a triple point. 

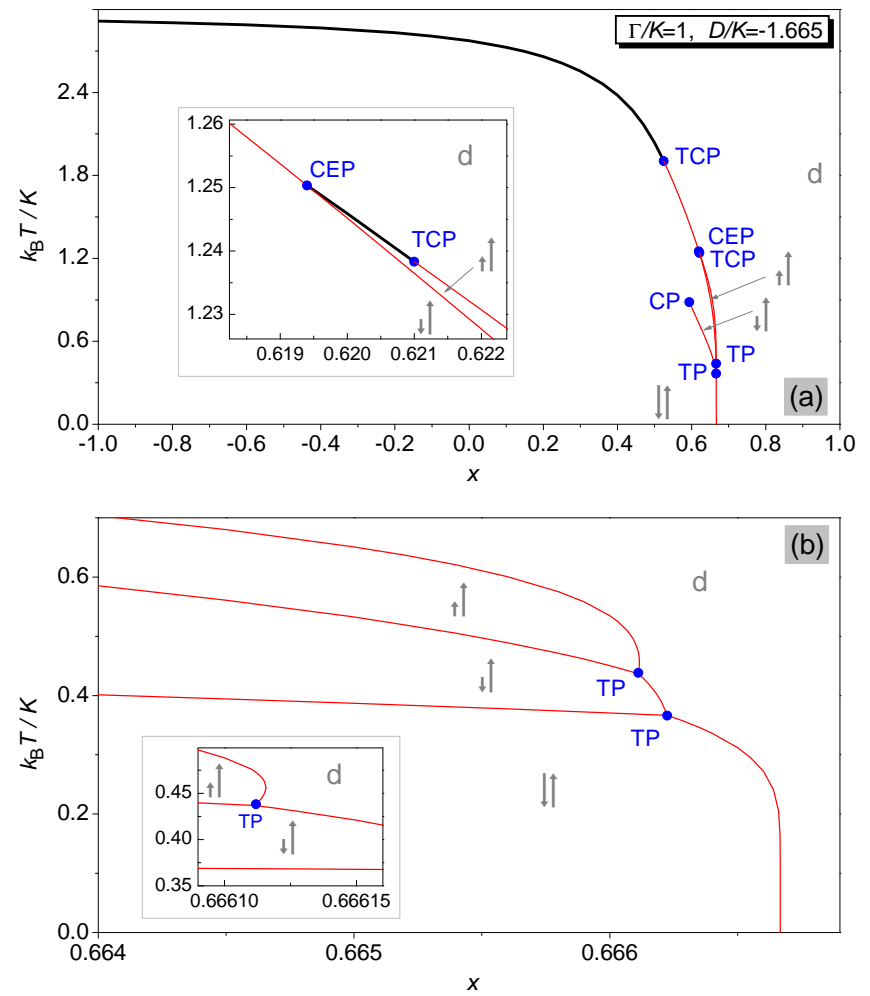

Figure 3: The $T$ vs $x$ phase diagram at $D / K=-1.665$ and $\Gamma / K=1$. A thick line indicates the phase transition between ordered and disordered phases of the second order. Thin lines indicate the first order phase transitions between ordered and disordered phases as well as between different ordered phases. The special points are tricritical points, a critical end point, a critical point and triple points. Enlarge parts of the phase diagram are presented in subfigure (b) and inserts. 

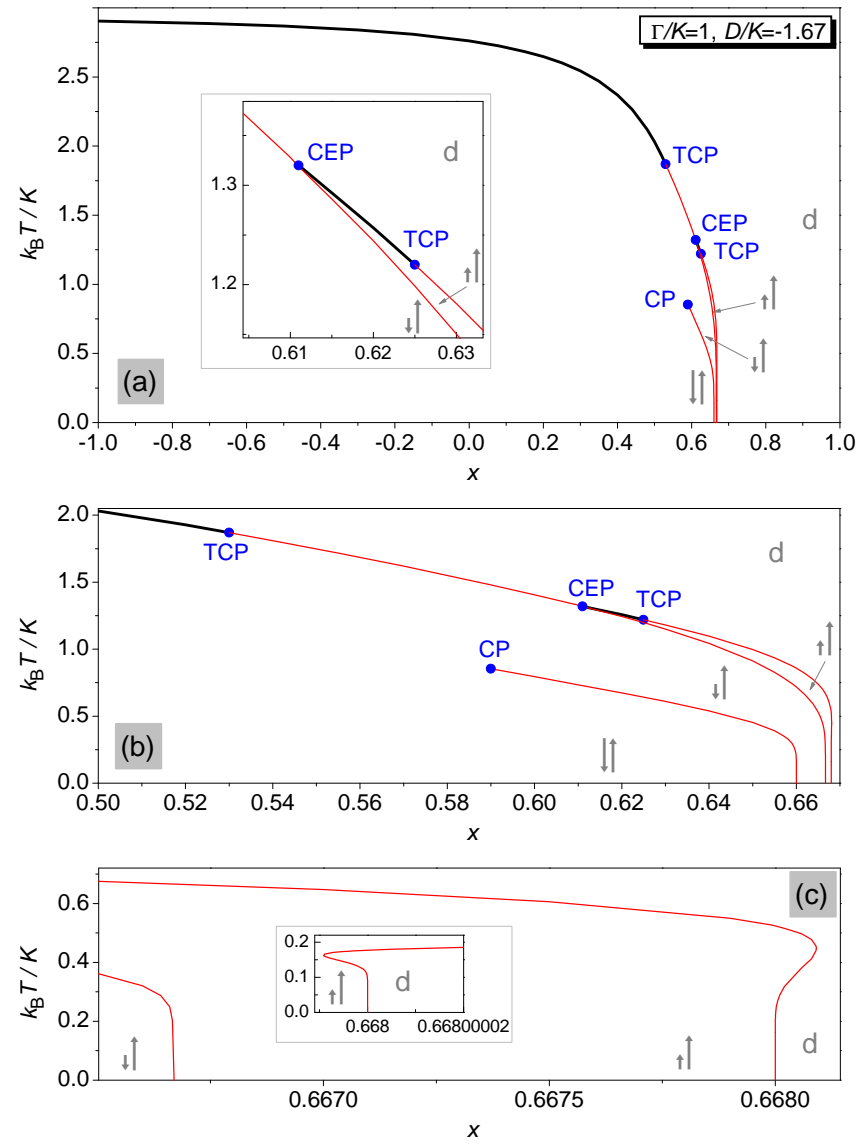

Figure 4: The same as in Fig. 3, but $D / K=-1.67$ and $\Gamma / K=1$.

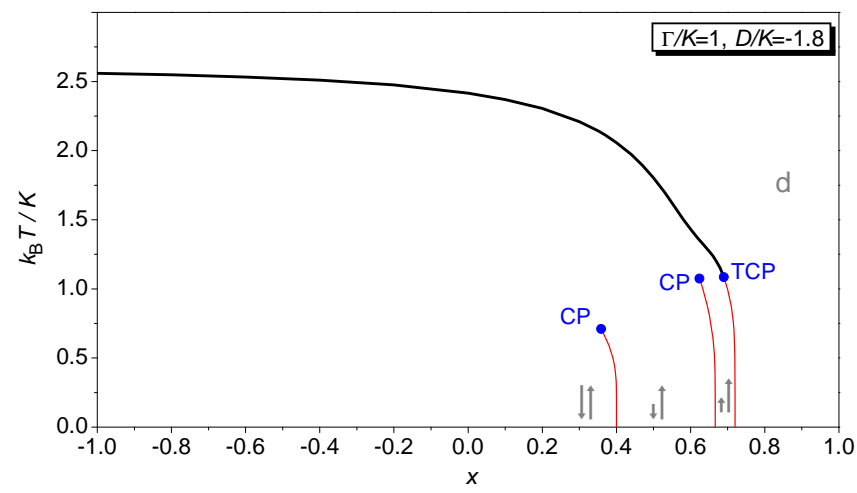

Figure 5: The same as in Fig. 3, but $D / K=-1.8$ and $\Gamma / K=1$. 


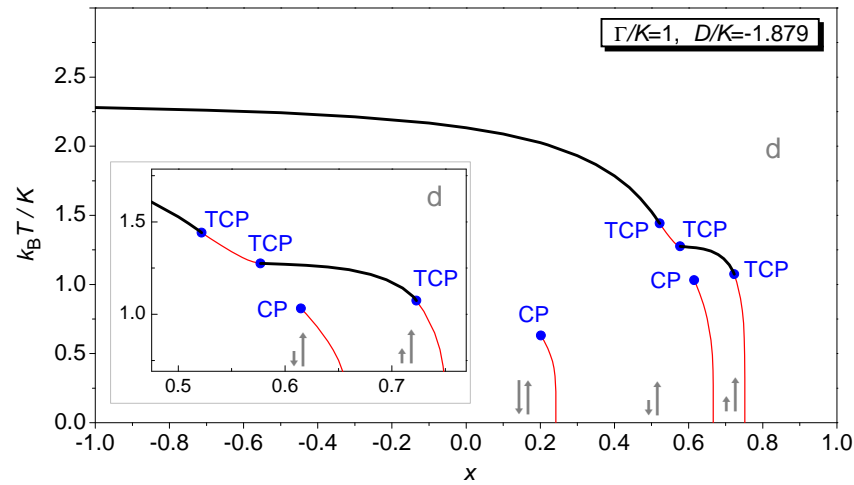

Figure 6: The same as in Fig. 3. but $D / K=-1.879$ and $\Gamma / K=1$.

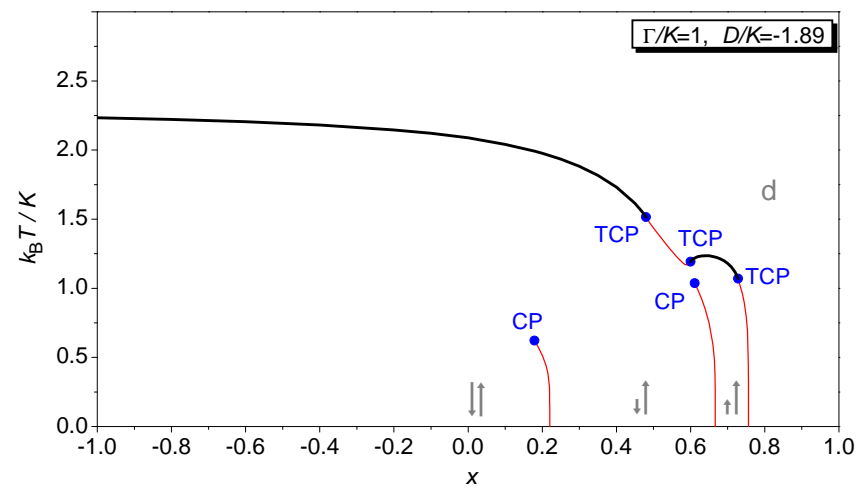

Figure 7: The same as in Fig. 3, but $D / K=-1.89$ and $\Gamma / K=1$.

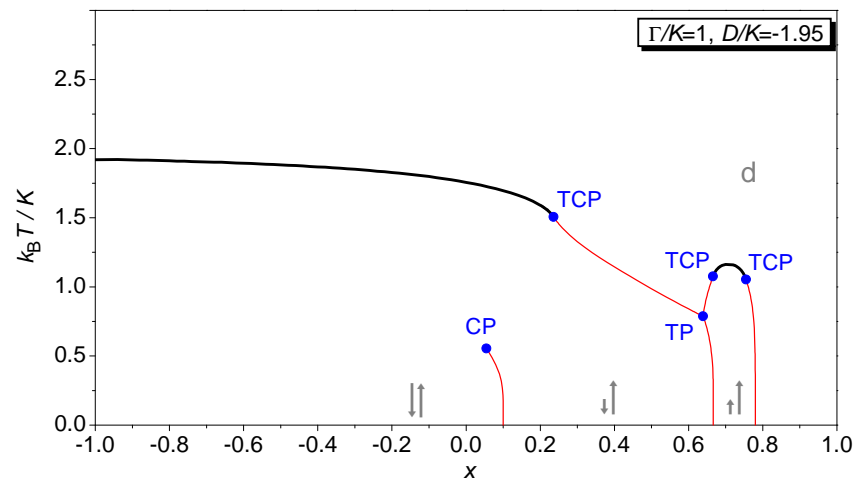

Figure 8: The same as in Fig. 3, but $D / K=-1.95$ and $\Gamma / K=1$. 


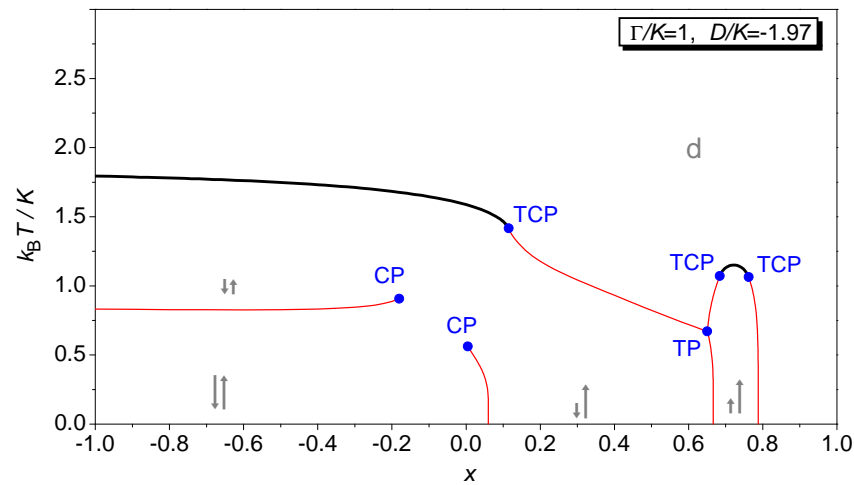

Figure 9: The same as in Fig. 3, but $D / K=-1.97$ and $\Gamma / K=1$.

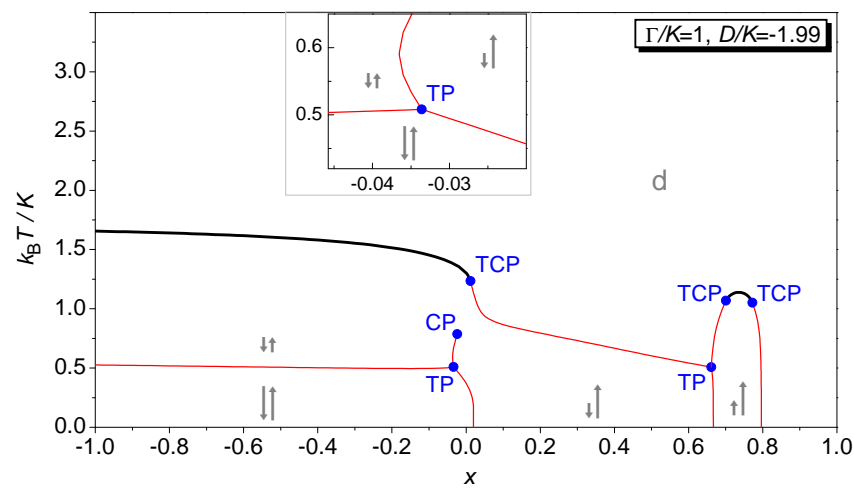

Figure 10: The same as in Fig. 3, but $D / K=-1.99$ and $\Gamma / K=1$. 

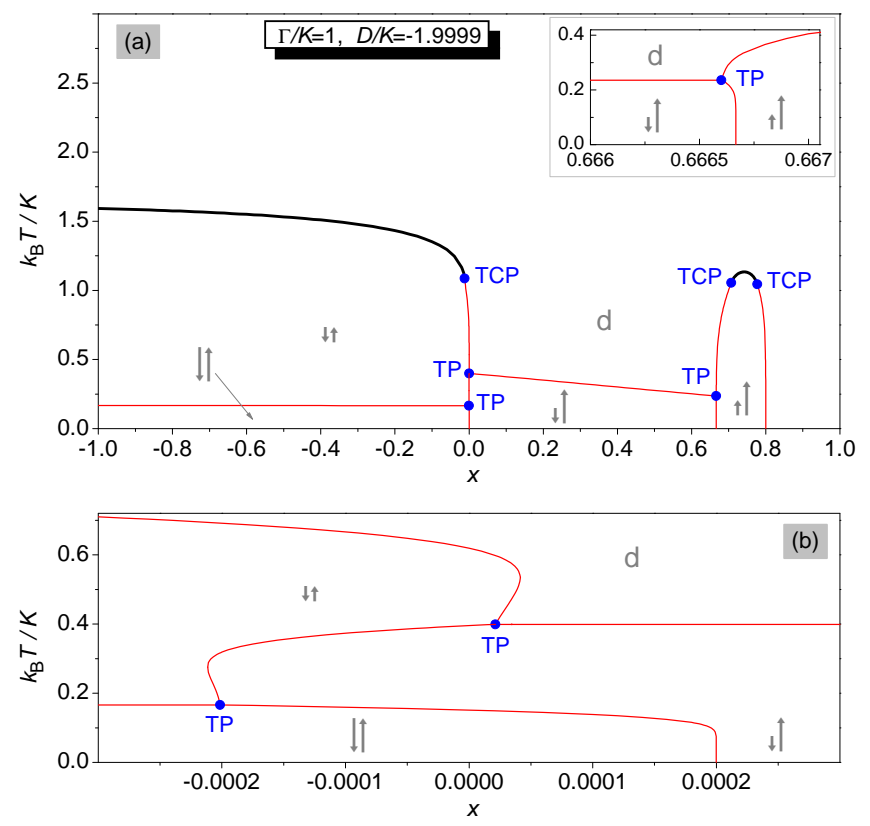

Figure 11: The same as in Fig. 3 but $D / K=-1.9999$ and $\Gamma / K=1$.

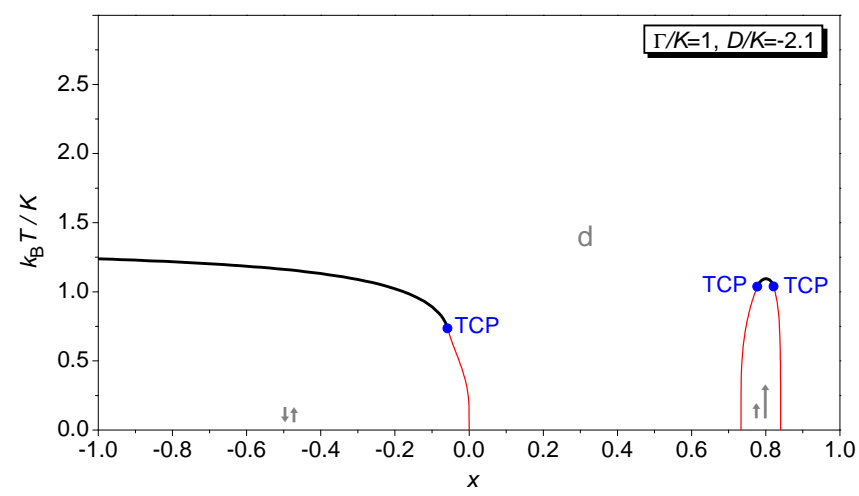

Figure 12: The same as in Fig. 3. but $D / K=-2.1$ and $\Gamma / K=1$. 


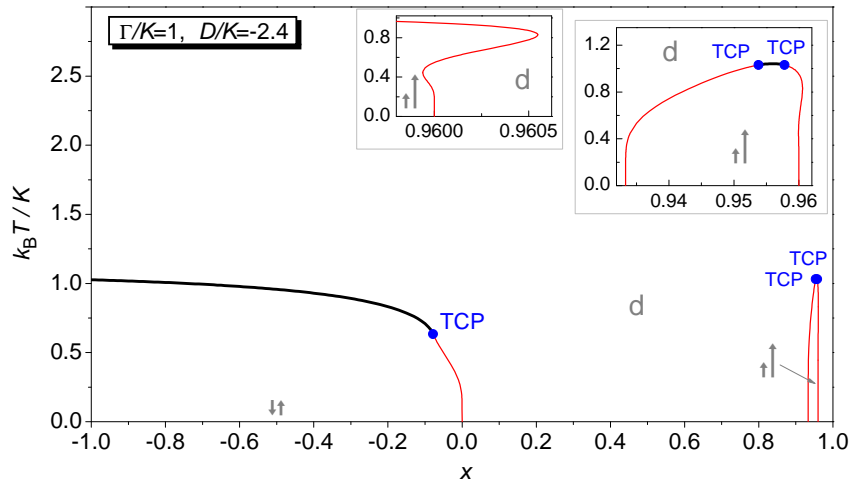

Figure 13: The same as in Fig. 3, but $D / K=-2.4$ and $\Gamma / K=1$.

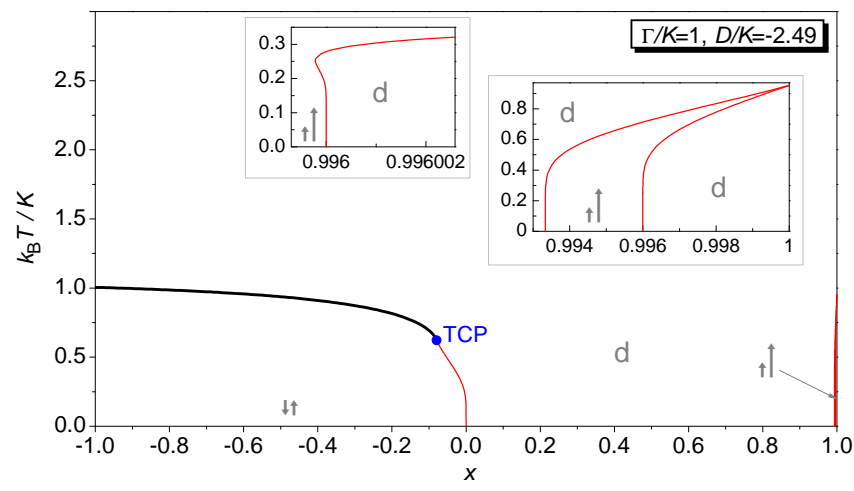

Figure 14: The same as in Fig. 3, but $D / K=-2.49$ and $\Gamma / K=1$.

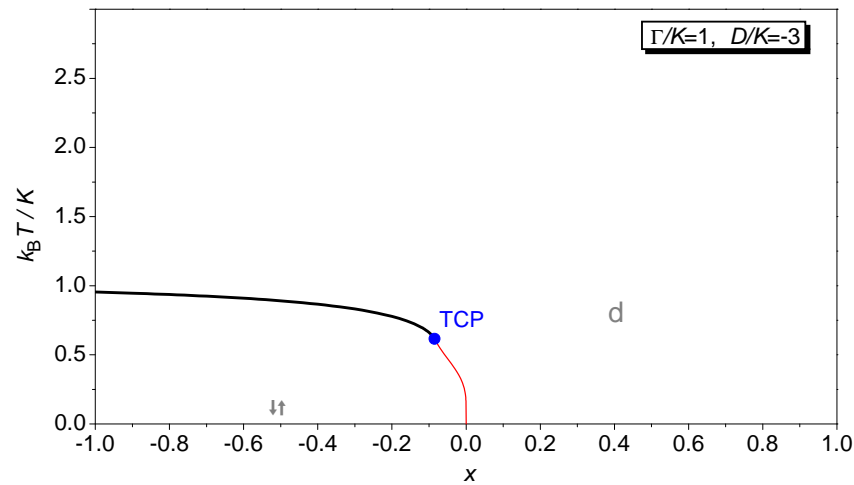

Figure 15: The same as in Fig. 3, but $D / K=-3$ and $\Gamma / K=1$. 


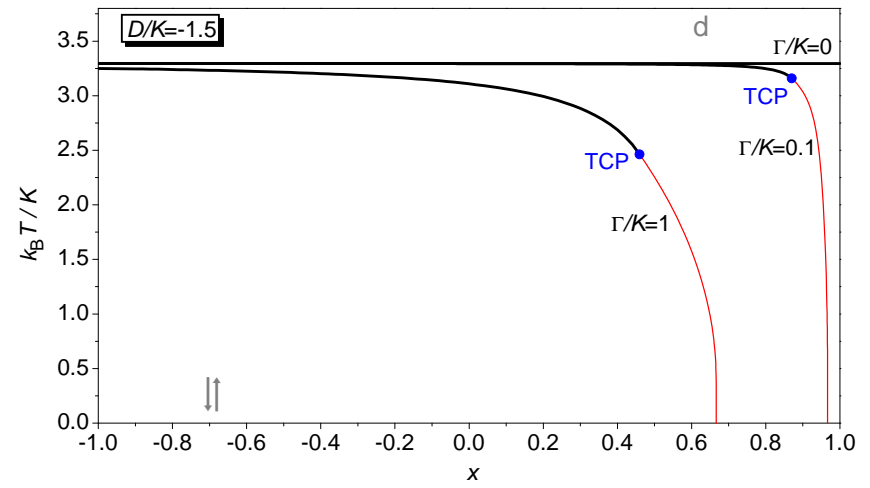

Figure 16: The same as in Fig. 3 , but $D / K=-1.5$ and $\Gamma / K=0.0,0.1,1.0$.

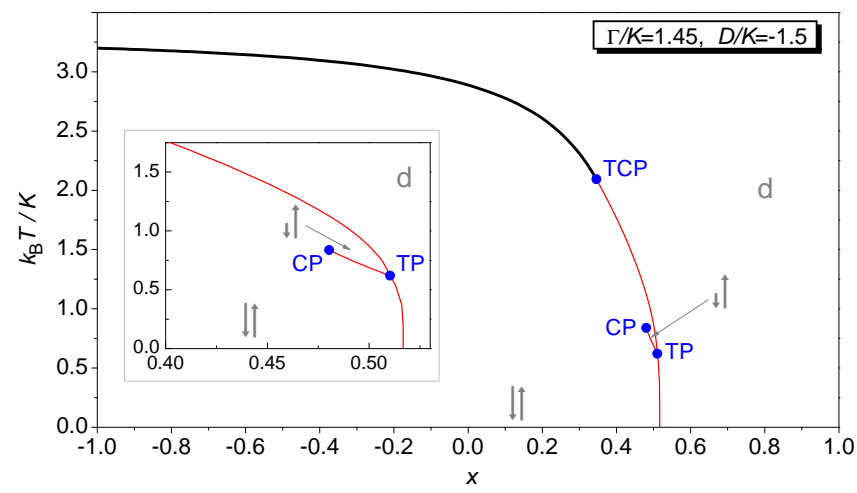

Figure 17: The same as in Fig. 3 , but $D / K=-1.5$ and $\Gamma / K=1.45$. 


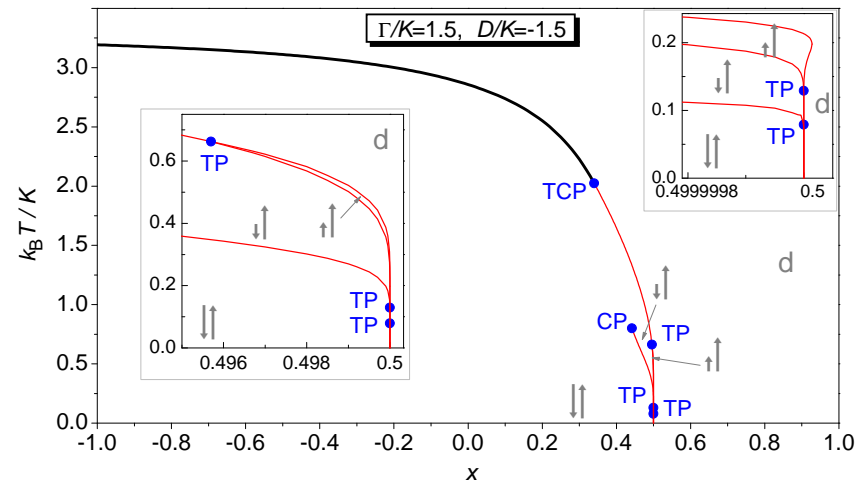

Figure 18: The same as in Fig. 3, but $D / K=-1.5$ and $\Gamma / K=1.5$.

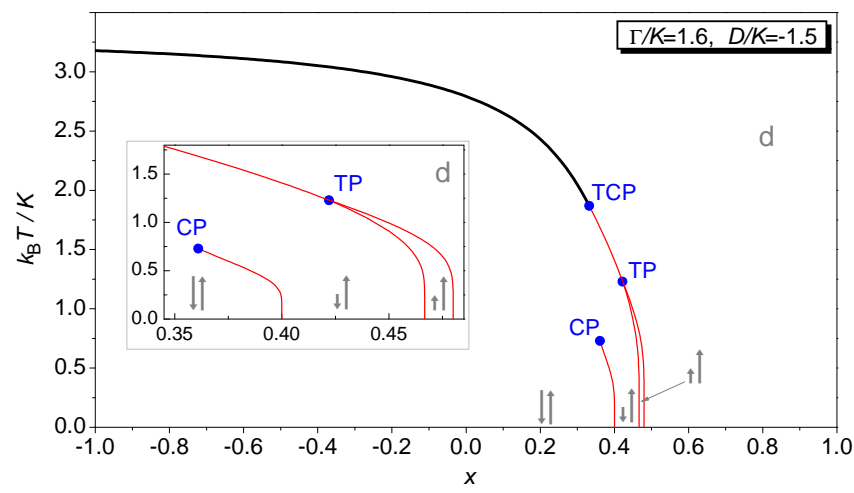

Figure 19: The same as in Fig. 3, but $D / K=-1.5$ and $\Gamma / K=1.6$. 


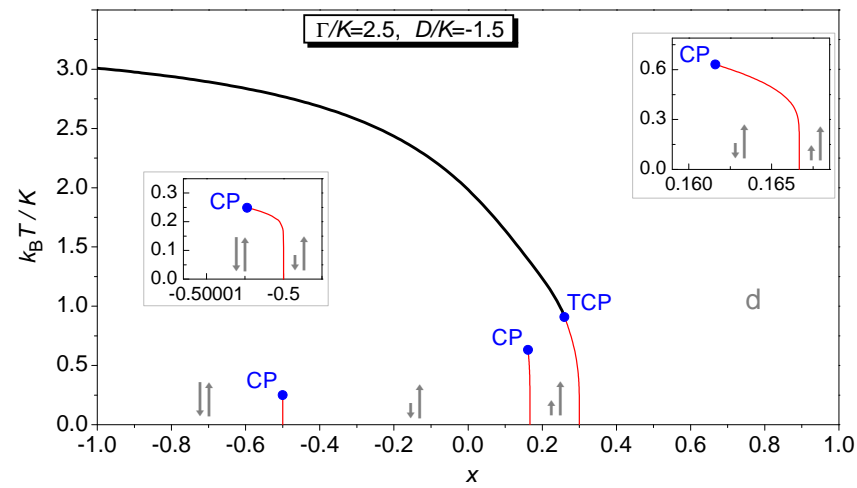

Figure 20: The same as in Fig. 3. but $D / K=-1.5$ and $\Gamma / K=2.5$.

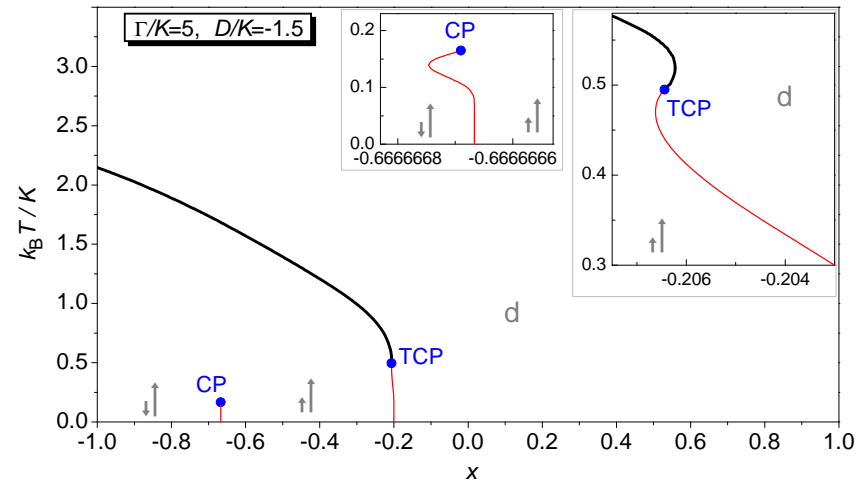

Figure 21: The same as in Fig. 3, but $D / K=-1.5$ and $\Gamma / K=5$. 


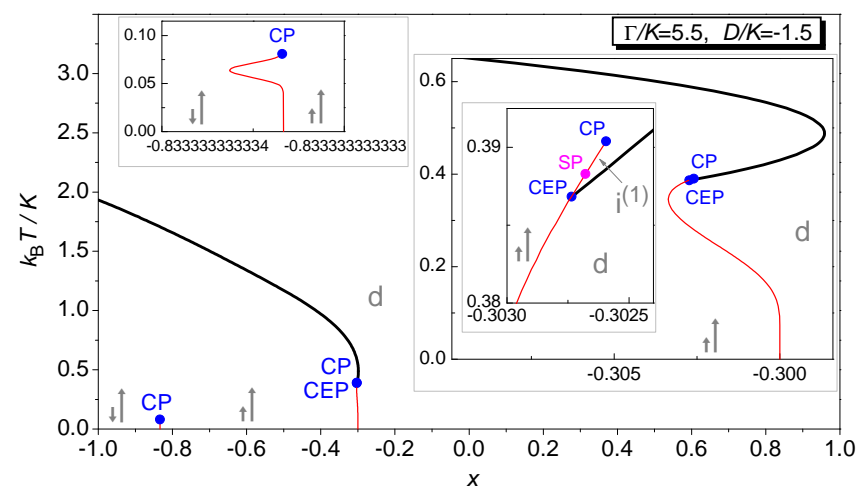

Figure 22: The $T$ vs $x$ phase diagram at $D / K=-1.5$ and $\Gamma / K=5.5$. A thick line indicates the phase transition between ordered and disordered phases of the second order. Thin lines indicate the first order phase transitions between ordered and disordered phases as well as between different ordered phases. The special points are a critical end point, critical points and SP.

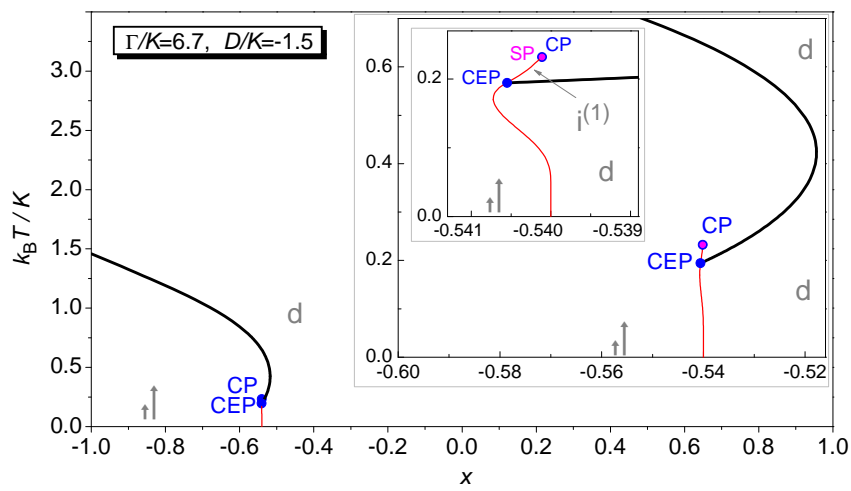

Figure 23: The same as in Fig. 22, but $D / K=-1.5$ and $\Gamma / K=6.7$. 


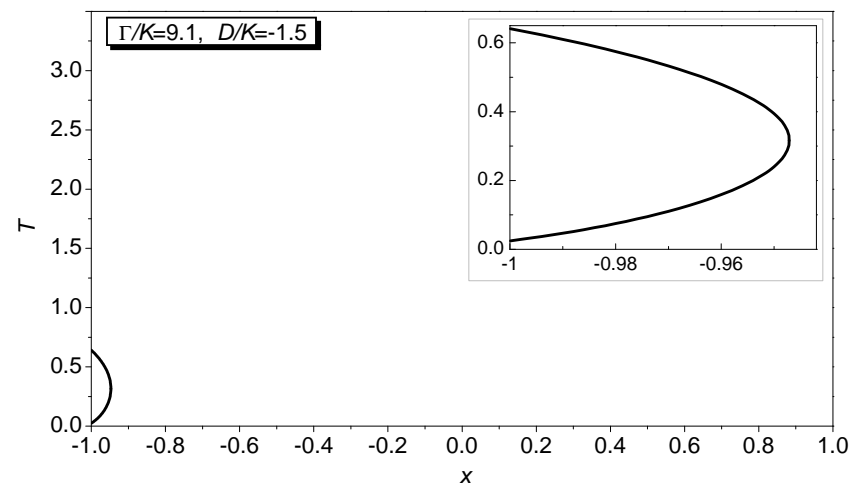

Figure 24: The same as in Fig. 22, but $D / K=-1.5$ and $\Gamma / K=9.1$.

\section{References}

[1] K. Takahashi, M. Tanaka, J. Phys. Soc. Japan 48 (1980) 1423.

[2] K. Kasono, I. Ono, Z. Phys. B - Condensed Matter, 88 (1992) 205.

[3] R. R. Netz, A. N. Berker, Phys. Rev. B 47 (1993) 15019.

[4] A. Bakchich, S. Bekhechi, A. Benyoussef, Physica A 210 (1994) 415.

[5] S. Bekhechi, A. Benyoussef, Phys. Rev. B 56 (1997) 13954.

[6] O. R. Baran, R. R. Levitskii, Phys. Stat. Sol. (b) 219 (2000) 357.

[7] O. R. Baran, R. R. Levitskii, Phys. Rev. B 65 (2002) 172407.

[8] R. R. Levitskii, O. R. Baran, B. M. Lisnii, Eur. Phys. J. B 50 (2006) 439.

[9] C. Ekiz, E. Albayrak, M. Keskin, J. Magn. Magn. Mater. 256 (2003) 311. 
[10] C. Ekiz, J. Magn. Magn. Mater. 284 (2004) 409.

[11] M. Keskin, M. Ali Pinar, A. Erdinç, O. Canko, Phys. Lett. A 353 (2006) 116.

[12] M. Keskin, M. Ali Pinar, A. Erdinç, O. Canko, Physica A 364 (2006) 263.

[13] J. Sivardiére, M. Blume, Phys. Rev. B 5 (1972) 1126.

[14] S. Krinsky, D. Mukamel, Phys. Rev. B 11 (1975) 399.

[15] D. Horváth, A. Orendáčová, M. Orendáč, M. Jaščur, B. Brutovský, A. Feher, Phys. Rev. B 60 (1999) 1167.

[16] A. Orendáčová, D. Horváth, M. Orendáč, E. Čižmár, M. Kačmár, V. Bondarenko, A.G. Anders, A. Feher, Phys. Rev. B 65 (2001) 014420.

[17] O. Baran, R. Levitskii, J. Magn. Magn. Mater. 324 (2012) 3778.

[18] F. C. Sá Baretto, O. F. De Alcantara Bonfim, Physica A 172 (1991) 378.

[19] A. Bakkali, M. Kerouad, M. Saber, Physica A 229 (1996) 563.

[20] T. Kaneyoshi, M. Jaščur, Phys. Lett. A 177 (1993) 172.

[21] J. W. Tucker, J. Magn. Magn. Mater. 214 (2000) 121.

[22] A. Bakchich, A. Bassir, A. Benyoussef, Physica A 195 (1993) 188.

[23] J. C. Xavier, F. C. Alcaraz, D. Penâ Lara, J. A. Plascak, Phys. Rev. B 57 (1998) 11575. 
[24] J. S. Smart, Effective field theories of magnetism, W.B. Saunders company, Philadelphia-London, 1996, p. 188.

[25] H. H. Chen, P. M. Levy, Phys. Rev. B 7 (1973) 4267.

[26] M. Blume, V. J. Emery, R.B. Griffiths, Phys. Rev. A 4 (1971) 1071.

[27] H. Yoshizawa, D. P. Belanger, Phys. Rev. B 30 (1984) 5220.

[28] J. A. Plascak, J. G. Moreira, F. C. sá Baretto, Phys. Lett. A 173 (1993) 360. 